\title{
Captura do caranguejo-uçá a partir do conhecimento tradicional: perfil socioeconômico e inovação da extração no estado do Rio de Janeiro, Brasil
}

Gathering of the mangrove crab from traditional knowledge: socioeconomic profile and innovation of extraction in Rio de Janeiro State, Brazil

\author{
Laura Helena de Oliveira Côrtes', Ana Paula Madeira Di Beneditto', Camilah Antunes Zappes"I \\ Universidade Estadual do Norte Fluminense. Campos dos Goytacazes, Rio de Janeiro, Brasil \\ "Universidade Federal Fluminense. Campos dos Goytacazes, Rio de Janeiro, Brasil
}

\begin{abstract}
Resumo: O presente estudo descreve uma inovação local ('ratoeira') utilizada na comunidade de Gargaú, costa norte do estado do Rio de Janeiro, para captura comercial do caranguejo-uçá [Ucides cordatus (Linnaeus, 1763)] no manguezal do estuário do rio Paraíba do Sul. A coleta de dados foi realizada entre abril e maio de 2016, a partir de 30 entrevistas etnográficas com catadores da comunidade e de observações em campo. Na área de estudo, a extração do caranguejo-uçá é realizada através dos métodos de 'braceamento', 'mão', 'redinha' e, mais recentemente, da 'ratoeira', que é uma inovação local para a captura de U. cordatus, utilizada com essa finalidade desde 2013. Esse artefato é uma armadilha que consiste de um alçapão confeccionado em plástico e madeira. Ela captura os animais vivos, permitindo a seleção de sexo e de tamanho dos caranguejos-uçá para comercialização. Os métodos não tradicionais de extração utilizados na região, como a 'ratoeira' e a 'redinha', devem estar associados a estratégias de manejo local, para minimizar seus possíveis impactos sobre a população capturada para fins comerciais.
\end{abstract}

Palavras-chave: Crustáceos. Armadilha. Catadores tradicionais. Norte fluminense.

\begin{abstract}
This study describes a local cultural innovation ('ratoeira' or rat-trap) used in the community of Gargaú, northern Rio de Janeiro State, for commercial gathering of the mangrove crab [Ucides cordatus (Linnaeus, 1763)] in mangrove forest of the Paraíba do Sul River estuary. Data collection was done between April and May 2016 from 30 ethnographic interviews conducted with crab gatherers and field observations. In the study area, mangrove crab gathering is done by 'redinha' or net method, 'braceamento' or arm method, and 'mão' or hand method, and more recently by 'ratoeira' or rat-trap. The 'ratoeira' is a local innovation to U. cordatus gathering that has been used since 2013. This artifact is a trap consisting of a trap door made of plastic and wood. The 'ratoeira' catches live animals, allowing sex and size selection of mangrove crabs for commercialization. The local non-traditional gathering methods of mangrove crabs, such as 'ratoeira' and 'redinha', should be considered with local management strategies to minimize their impact on population harvested for commercial purposes.
\end{abstract}

Keywords: Crustaceans. Trap. Traditional gatherers. Northern fluminense.

CÔRTES, L. H. O., A. P. M. DI BENEDITTO \& C. A. ZAPPES, 2018. Captura do caranguejo-uçá a partir do conhecimento tradicional: perfil socioeconômico e inovação da extração no estado do Rio de Janeiro, Brasil. Boletim do Museu Paraense Emílio Goeldi. Ciências Naturais 13(1): 45-55.

Autora para correspondência: Camilah Antunes Zappes. Universidade Federal Fluminense. Programa de Pós-Graduação em Geografia. Instituto de Ciências da Sociedade e Desenvolvimento Regional. Laboratório de Geografia Física. Rua José do Patrocínio, 71 - Centro. Campos dos Goytacazes, RJ, Brasil. CEP 28010-385 (camilahaz@yahoo.com.br).

Recebido em 10/10/2017

Aprovado em 26/03/2018

Responsabilidade editorial: Fernando da Silva Carvalho Filho

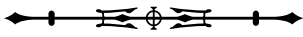




\section{INTRODUÇÃO}

Sociedades humanas e animais mantêm uma relação ancestral, construída ao longo de milênios, o que permitiu o desenvolvimento dos modos de uso da fauna local por comunidades tradicionais (Alves, 2012; Alves \& Souto, 2015). Pesquisas etnozoológicas são geralmente baseadas nas relações que envolvem questões de conservação, de socioeconomia e de cultura (Alves, 2012). Tais estudos mostram-se relevantes, pois permitem aos pesquisadores compreender a relação do homem com a fauna e, a partir disso, possibilita o trabalho com ações de conservação (Alves, 2012). No Brasil, pesquisas que envolvem comunidades tradicionais e recursos faunísticos de manguezal, como os caranguejos, são realizadas em grande parte da costa do país (Alves, 2012; Alves \& Nishida, 2002; Nordiet al., 2009; Côrtes et al., 2014a; Nascimento et al., 2016).

Os manguezais são ecossistemas costeiros que ocorrem em regiões tropicais e temperadas quentes, dominados por espécies lenhosas, sendo caracterizados pela elevada amplitude de marés e por disponibilidade de nutrientes (Feller et al., 2010). Esses ecossistemas ocupam cerca de $150.000 \mathrm{~km}^{2}$ em todo mundo e possuem função econômica associada ao fornecimento de alimentos, de madeira, de corantes e de medicamentos (Feller et al., 2010; Spalding et al., 2010; Giri et al., 2011).

O caranguejo-uçá [Ucides cordatus (Linnaeus, 1763)] é a principal espécie de crustáceo extraída comercialmente nos manguezais brasileiros (Alves \& Nishida, 2002; Fiscarelli \& Pinheiro, 2002; Capistrano \& Lopes, 2012). A sua captura ocorre ao longo de toda costa, com cadeias produtivas estruturadas em torno de sua comercialização (Dias-Neto, 2011; Côrtes et al., 2014b; Nascimento et al., 2016). Com o aumento do número de catadores nos manguezais brasileiros, vem ocorrendo a substituição de métodos de captura tradicionais por não tradicionais e mais eficientes. Isso leva à redução dos estoques de caranguejouçá, interferindo negativamente sobre as comunidades que dependem dessa atividade extrativa para subsistência (Nordi et al., 2009; Nascimento et al., 2012).
A portaria n. 52, de 30 setembro de 2003, do Instituto Brasileiro do Meio Ambiente e dos Recursos Naturais Renováveis (IBAMA) regulamenta a extração do caranguejo-uçá nas regiões Sul e Sudeste do Brasil (18 $\mathrm{S}-28^{\circ} \mathrm{S}$ ). Esta portaria estabelece o período de defeso anual da espécie e a largura mínima de carapaça dos animais capturados, proibindo a captura e a comercialização de fêmeas ovígeras e de partes do corpo do animal durante todo ano, bem como a utilização de quaisquer artefatos, armadilhas e produtos químicos para a captura do caranguejo-uçá em qualquer época do ano (Brasil, 2003a).

O estado do Rio de Janeiro produz 46 t/ano de caranguejo-uçá, representando 45\% da produção total do Sudeste do Brasil (Dias-Neto, 2011). No norte do estado, a captura da espécie para fins comerciais é praticada pelas comunidades de Gargaú e Atafona, com maior representatividade para a primeira comunidade (Côrtes et al., 2014a, 2014b). Ambas situam-se no entorno do manguezal do estuário do rio Paraíba do Sul, que tem área aproximada de 800 ha (Bernini, 2008). Os métodos empregados na captura desta espécie pelos catadores de Gargaú são 'braceamento', 'mão' e 'redinha' (Côrtes et al., 2014a). Em áreas do Nordeste do país, são descritas técnicas de captura por armadilhas como 'redinha' e 'ratoeira' (Alves et al., 2005; Barboza et al., 2008; Nascimento et al., 2011, 2016, 2017). No entanto, para o norte do estado do Rio de Janeiro esta é a primeira descrição do desenvolvimento de uma inovação local voltada à captura comercial da espécie.

Assim, o objetivo do presente estudo é descrever uma inovação local ('ratoeira') utilizada na comunidade de Gargaú, costa norte do estado do Rio de Janeiro, para a captura comercial do caranguejo-uçá [Ucides cordatus (Linnaeus, 1753)] no manguezal do estuário do rio Paraíba do Sul.

\section{MATERIAL E MÉTODOS}

\section{ÁREA DE ESTUDO}

O estudo foi desenvolvido na comunidade de Gargaú ( $21^{\circ} 36^{\prime}$ S; $41^{\circ} 03^{\prime}$ W), município de São Francisco de 
Itabapoana, localizado no estuário secundário do rio Paraíba do Sul (Figura 1). Nesta comunidade está sediada a colônia de pescadores Z-1, com 1.375 associados registrados como pescadores e 50 como catadores de caranguejo. A extração do caranguejo-uçá é exercida como principal atividade econômica para a maior parte dos catadores, mas também pode ser fonte de renda complementar (Côrtes et al., 2014b).

\section{COLETA E ANÁLISE DE DADOS}

Entre os meses de abril e maio de 2016, foram coletadas informações socioeconômicas dos catadores e relatos sobre os métodos de extração do caranguejo-uçá na região por meio de 30 entrevistas etnográficas (o que representa 60\% dos catadores em atuação na região) e de observações em campo. Para a realização das entrevistas, foi utilizado questionário-padrão, composto por questões abertas, subdividido em duas categorias: 1) perfil socioeconômico dos entrevistados (sexo; idade; renda mensal per capita e familiar derivada da comercialização do caranguejo-uçá; proporção de renda per capita e domiciliar derivada da comercialização do caranguejouçá; e recebimento de benefícios sociais) e 2) extração do caranguejo-uçá (incluindo os métodos de captura utilizados, modo de utilização da 'ratoeira', seu custo de produção e as vantagens de sua utilização).

A coleta de dados qualitativos foi realizada por meio de amostragem intencional, na qual os participantes da pesquisa foram selecionados de acordo com critérios pré-determinados (Patton, 1990): ser catador de caranguejouçá e atuar no manguezal do estuário do rio Paraíba do Sul.

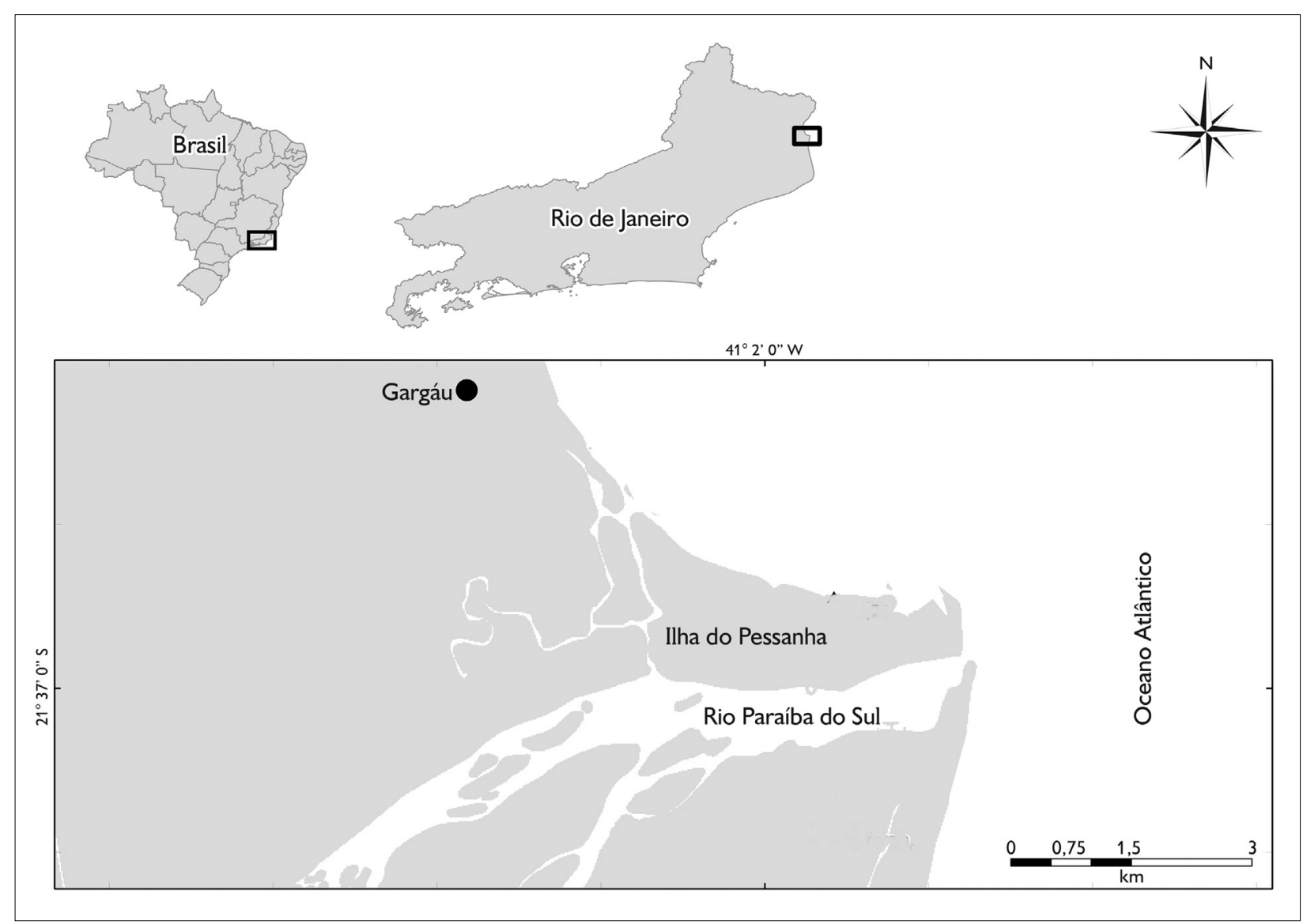

Figura 1. Localização da comunidade de Gargaú e do estuário do rio Paraíba do Sul, norte do estado do Rio de Janeiro.

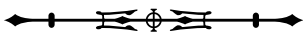


A seleção do primeiro entrevistado foi realizada pela amostragem oportunista de um membro da comunidade que havia colaborado em pesquisas anteriores (Côrtes et al., 2014a, 2014b). Os demais participantes também foram selecionados por abordagem oportunista ou por meio do método 'bola de neve', em que um entrevistado indica outros potenciais respondentes na comunidade, formando uma rede de informações (Bailey, 1982).

Para a realização das entrevistas, foi solicitada a emissão de anuência prévia à presidente da colônia de pescadores Z-1, representante legal desta categoria profissional na região. $\bigcirc$ documento autoriza a condução de estudos que tratam do conhecimento tradicional de comunidades (Azevedo, 2005; Brasil, 2015). Antes de cada entrevista, os objetivos da pesquisa e a condição de anonimato foram informados aos entrevistados, os quais foram consultados quanto ao aceite em participar do estudo (Librett \& Perrone, 2010).

\section{RESULTADOS E DISCUSSÃO}

Entre os 30 entrevistados na comunidade, 53\% ( $n=$ 16) pertencem ao sexo masculino e $47 \%(n=14)$, ao sexo feminino, com idades entre 20 e 67 anos. A faixa etária mais representativa variou entre 41 e 50 anos (40\%, n = 12) (Tabela 1). Em outras áreas de manguezal do país, a predominância de catadores na extração do caranguejouçá é de homens, devido à característica insalubre da atividade, sendo a participação da mulher restrita apenas ao processamento do produto (Fiscarelli \& Pinheiro, 2002; Alves \& Nishida, 2003; Barboza et al., 2008; Nascimento et al., 2011, 2016; Walter et al., 2012). De acordo com Vergara Filho \& Pereira Filho (1995) e Côrtes et al. (2014b), a extração de $U$. cordatus no norte do estado do Rio de Janeiro era realizada com predominância do sexo feminino, representando uma peculiaridade da região.

No presente estudo, homens e mulheres atuam em proporções semelhantes nesta atividade, o que demonstra uma modificação no perfil dos catadores de caranguejo-uçá entre os anos de 2014 e 2016.
Tabela 1. Faixa etária dos catadores de caranguejo-uçá entrevistados na comunidade de Gargaú, costa norte do estado do Rio de Janeiro.

\begin{tabular}{c|c|c}
\hline Faixa etária & Número de entrevistados & $\%$ \\
\hline $20-30$ & 2 & 7 \\
\hline $31-40$ & 6 & 20 \\
\hline $41-50$ & 12 & 40 \\
\hline $51-60$ & 9 & 30 \\
\hline Acima de 60 & 1 & 3 \\
\hline Total & 30 & 100 \\
\hline
\end{tabular}

Em geral, a pesca artesanal praticada na região é realizada com predominância do sexo masculino (Zappes et al., 2016). Assim, o aumento da proporção de homens nesta atividade demonstra a necessidade de incremento da renda mensal por meio da diversificação das atividades pesqueiras, levando-os a participarem de tarefas que antes eram praticadas prioritariamente pelas mulheres da comunidade (Côrtes et al., 2014b).

O intervalo de faixa etária dos catadores demonstra a renovação da população que atua na extração do caranguejo-uçá na comunidade, visto que, em 2014, a idade destes trabalhadores em atuação variava entre 23 e 76 anos (Côrtes et al., 2014a). A idade inicial na prática extrativa foi semelhante à encontrada por Zappes et al. (2016) para pescadores artesanais do norte do estado do Rio de Janeiro (22 anos). Isso demonstra a transmissão familiar de profissões relacionadas à pesca, prática comum em comunidades tradicionais (Diegues, 2000; Clauzet et al., 2005; Martins, 2008).

Os catadores da comunidade são caracterizados por apresentarem baixa escolaridade, com ensino fundamental incompleto. Esse padrão é semelhante ao encontrado em outras regiões brasileiras (Fiscarelli \& Pinheiro, 2002; Alves \& Nishida, 2003). O baixo nível de escolaridade e a carência de oportunidades de empregos em outros setores da economia local resultam em uma relação de dependência econômica da comunidade com relação às atividades pesqueiras, dificultando a organização administrativa e socioeconômica do local (Alves \& Nishida, 
2003; Silva et al., 2007; Alencar \& Maia, 2011; Côrtes et al., 2014b). Essa dependência econômica pode induzir a uma pressão ambiental sobre o ecossistema de manguezal e, consequentemente, sobre $U$. cordatus. Ainda, o reduzido ganho econômico conquistado torna esta atividade tradicional inviável economicamente (Nascimento et al., 2016). Apesar dessa dependência em relação à atividade, filhos de famílias que vivem de recursos pesqueiros não têm escolhido seguir a profissão de pescador/catador artesanal dos pais, devido às condições de trabalho e ao baixo retorno da atividade (Martins, 2008; Oliveira et al., 2016; Zappes et al., 2016).

A renda média mensal per capita estimada a partir dos relatos dos catadores da comunidade é de aproximadamente $R \$ 790,00$. Já a renda mensal domiciliar é, em média, de $R \$ 920,00$, e inclui o rendimento de todos os habitantes economicamente ativos no domicílio. No entanto, alguns entrevistados afirmaram ser os únicos provedores da casa. A renda mensal média oriunda da comercialização do caranguejo-uçá é de $R \$ 613,00$, o que equivale a $78 \%$ da renda mensal per capita e a $67 \%$ da renda mensal domiciliar.

A renda mensal per capita domiciliar relatada pelos catadores é inferior às médias nacional $(R \$ 1.113,00) \mathrm{e}$ estadual (R\$1.285,00) (IBGE, 2015). Além disso, este dado para a área urbana do município de São Francisco de Itabapoana é de $\mathrm{R} \$ 1.408,00$ (IBGE, 2016). Dessa forma, o rendimento obtido com a extração do caranguejo-uçá equivale a $50 \%$ das rendas nacional e estadual, e a $44 \%$ da renda per capita do município. Isso demonstra a baixa remuneração da atividade e a necessidade de fontes alternativas de renda para a manutenção dos catadores e de seus familiares (Alves et al., 2005). Apesar da extração do caranguejo-uçá ser pouco lucrativa para os trabalhadores, que ainda são marginalizados, por outro lado, o recurso que eles capturam é importante para a culinária do Brasil e apresenta valores elevados na comercialização final (Alves et al., 2005). Isso demonstra uma discrepância comum na cadeia produtiva relacionada às atividades tradicionais, em que o produtor/explorador obtêm pequena renda, enquanto o consumidor paga valores elevados pelo produto final.

A forte dependência econômica per capita e domiciliar da comunidade estimula a renovação da população de catadores em Gargaú. De acordo com Côrtes et al. (2014b), a renda mensal estimada para os catadores locais com a extração do caranguejo-uçá era de aproximadamente $\mathrm{R} \$$ 678,00 (valor referente ao salário mínimo nacional vigente em 2014). Esse valor é superior ao obtido no presente estudo e demonstra uma redução no retorno financeiro da atividade ao longo do tempo. A renda mensal dos catadores de caranguejo em outras comunidades extrativas do Brasil é de cerca de um salário mínimo nacional (Barboza et al., 2008; Santa-Fé \& Araújo, 2013). Mesmo assim, deve ser observado o período de realização destes estudos e considerada a possibilidade de que o mesmo padrão de diminuição de renda obtida com a atividade pode estar acontecendo ao longo do país, não sendo um fator pontual da região. Apesar do baixo rendimento mensal obtido, a maior parte dos catadores entrevistados está satisfeita e interessada em permanecer na atividade, o que pode estar relacionado à baixa escolaridade, induzindo à falsa sensação de que a extração do caranguejo é uma atividade satisfatória.

Nove entrevistados (27\%) afirmaram receber benefícios sociais concedidos pelo programa Bolsa Família, do governo federal, no valor de $\mathrm{R} \$ 77,00$ mensais por família. O programa foi instituído pelo governo através de lei n. 10.836, publicada em 9 de janeiro de 2004, e tem como finalidade promover o alívio da pobreza, por meio da transferência unificada de renda a famílias em situação de 'pobreza' ou de 'extrema pobreza' (Brasil, 2004). As famílias classificadas em situação de 'pobreza' apresentam renda per capita mensal de até $R \$ 170,00$, enquanto a renda individual de famílias em 'extrema pobreza' é de até $\mathrm{R} \$ 85,00$ (IBGE, 2016). Os entrevistados da comunidade recebem de acordo com a categoria 'benefício básico', segundo o 
decreto n. 8.232/2014, em que $R \$ 77,00$ mensais são destinados às unidades familiares de 'extrema pobreza', independente do número de habitantes ou da presença de crianças e de adolescentes no domićlio (Brasil, 2014). A partir de julho de 2016, período posterior ao estudo em questão, o valor do benefício básico do programa foi reajustado para $R \$ 85,00$, por meio do decreto $n$. 8.794, de 29 de junho de 2016 (Brasil, 2016), o que elevou o valor da renda mensal domiciliar em $\mathrm{R} \$ 8,00$.

Na comunidade de Gargaú, a extração do caranguejo-uçá é realizada por meio dos métodos 'redinha' ( $n=26 ; 45 \%)$, 'mão' ( $n=18 ; 31 \%)$, 'braceamento' ( $n=11 ; 19 \%)$ e, mais recentemente, 'ratoeira' ( $n=3$; 5\%). Cada catador entrevistado pode utilizar mais de um método de captura, o que explica o maior número de respostas $(n=58)$ em relação ao número total de entrevistados ( $n=30)$. Os métodos 'braceamento', 'mão' e 'redinha', praticados na região, foram detalhados por Côrtes et al. (2014a). Os dados do presente estudo corroboram o levantamento realizado por Côrtes et al. (2014a), indicando o predomínio da 'redinha' sobre os demais métodos de captura. A utilização da 'redinha' é relatada desde a década de 1990 na região estudada (Vergara Filho \& Pereira Filho, 1995), sendo também descrita em outras áreas do país (Nascimento et al., 2011, 2016, 2017).

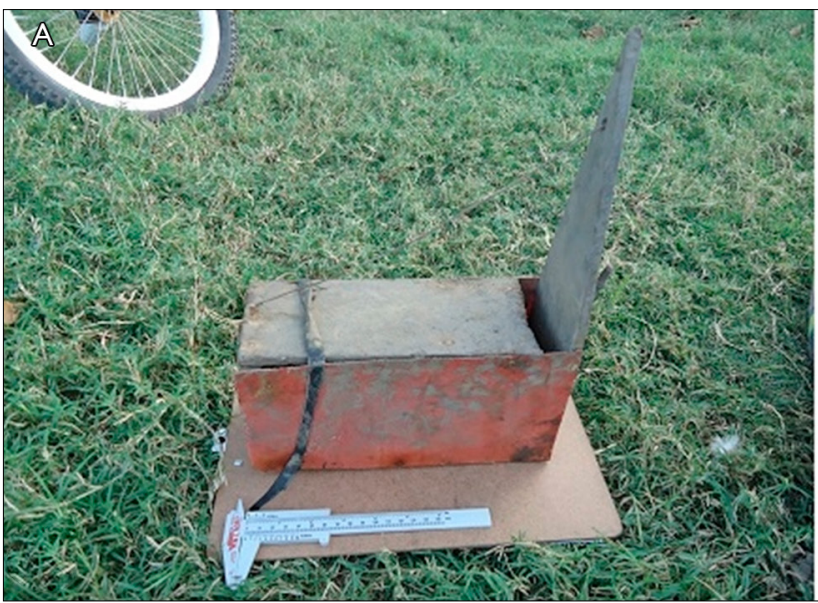

A 'ratoeira' é uma armadilha já utilizada na captura do caranguejo-uçá em outras áreas de manguezal, ao longo da costa brasileira (Ivo \& Gesteira, 1999; Nascimento et al., 2012, 2011), mas representa uma inovação local como método direcionado à captura comercial da espécie no manguezal do estuário do rio Paraíba do Sul, introduzida em 2013 e utilizada desde então. Originalmente, esse método era utilizado na comunidade de Gargaú somente para captura do guaiamum (Cardisoma guanhumi Latreille, 1825), mas a extração comercial desta espécie é realizada de modo pontual (Côrtes et al., 2014a), sofrendo adaptações para a captura do caranguejo-uçá na região.

A 'ratoeira' consiste de um alçapão em formato de paralelepípedo, confeccionado a partir de pedaços de plástico rígido, provenientes de caixas utilizadas para armazenar gelo e pescado, madeira e uma faixa de borracha, produzida a partir de câmara de pneumático. A armadilha mede $12 \mathrm{~cm}$ de altura, $10,5 \mathrm{~cm}$ de largura e $26 \mathrm{~cm}$ de profundidade (Figura 2). Em geral, ela é posicionada no solo do manguezal, às proximidades de galerias, identificadas pelos catadores como pertencentes aos machos de grande porte da espécie, fechando-se a partir do toque do animal na isca atrativa, aprisionando-o ainda vivo em seu interior. Cubos de abacaxi e de folhas da vegetação de manguezal são colocados no interior do alçapão como iscas, atraindo

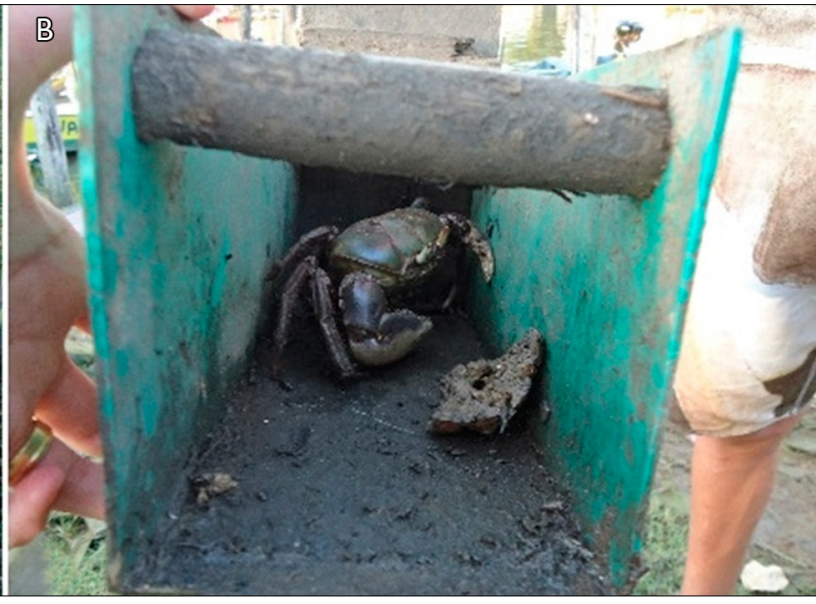

Figura 2. 'Ratoeira' utilizada pelos catadores da comunidade de Gargaú para captura do caranguejo-uçá (A) e um exemplar da espécie capturado (B). Fotos: Laura Helena de Oliveira Côrtes.

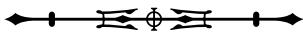


o caranguejo-uçá para fora de sua galeria e para o interior da 'ratoeira'. Segundo os entrevistados, o odor do abacaxi é o mais atrativo para o animal. Outros frutos, como laranja e goiaba, já foram testados como isca pelos catadores, mas sem sucesso na atração do animal.

Cada catador possui entre 50 e 180 'ratoeiras', que são posicionadas simultaneamente no manguezal, permanecendo assim durante todos os dias da semana. As armadilhas são inspecionadas diariamente in situ, para reposição das iscas e coleta dos caranguejos-uçá capturados. A retirada dos artefatos do manguezal ocorre apenas para a realização de reparos que, quando necessários, são feitos na sede da comunidade.

O percentual de catadores que atualmente faz uso deste método na região é baixo. Cerca de 300 'ratoeiras' são utilizadas em simultâneo no manguezal no estuário do rio Paraíba do Sul (800 ha), representando 2,6 'ratoeiras'/ha. Quanto maior o número de armadilhas, maior é o esforço e o sucesso de captura do catador a cada dia de trabalho. No entanto, isso também representa maior pressão de exploração sobre o estoque local do caranguejo-uçá. Segundo Alves et al. (2005), a atividade de extração é uma das principais interferências antrópicas sobre $U$. cordatus. Diante disso, a utilização deste método requer fiscalização e acompanhamento regular, especialmente se houver aumento no número de catadores.

De acordo com os catadores entrevistados, a armadilha captura os animais principalmente durante a noite, devido ao maior período de atividade da espécie sobre o solo do manguezal, e pode ser utilizada ao longo de todo ano. Nesse caso, as 'ratoeiras' são vistoriadas e os caranguejos-uçá capturados são recolhidos no dia seguinte. Características operacionais semelhantes foram reportadas por Botelho et al. (2000), Magalhães et al. (2011) e Firmo et al. (2012), nos estados de Pernambuco e da Bahia. Furtos das armadilhas ou dos caranguejos-uçá capturados são relatados pelos catadores da comunidade de Gargaú, e isso decorre provavelmente do longo tempo de permanência delas no solo do manguezal sem a supervisão direta dos catadores.
Esse tipo de armadilha é permitido para a captura do guaiamum, sendo utilizado com essa finalidade em diversas comunidades tradicionais ao longo da costa brasileira (Brasil, 2003b; Dias-Neto, 2011; Firmo et al., 2012). No entanto, sua utilização é proibida para a captura comercial do caranguejo-uçá (Brasil, 2003a). Apesar disso, comunidades de catadores de várias regiões do Brasil também fazem uso deste artefato na captura desta espécie (e.g. , Pernambuco: Botelho et al., 2000; Ceará: Carvalho \& Igarashi, 2009; e Bahia: Magalhães et al., 2011).

$O$ custo de cada 'ratoeira' varia entre $R \$ 12,00$ e $R \$ 15,00$, mas a armadilha também pode ser confeccionada pelo próprio catador a partir de materiais disponíveis em sua residência ou de materiais de descarte, sem custo nenhum ou a um custo mínimo. Considerando isso e a quantidade de 'ratoeiras' utilizadas pelo catador (50 a 180), estima-se um investimento inicial de até $\mathrm{R} \$ 2.700,00$ para a execução da atividade extrativa do caranguejo-uçá a partir desse método de captura, o qual chega a ser seis vezes superior ao necessário para a utilização da 'redinha', que é o principal método de captura desta espécie no manguezal do estuário do rio Paraíba do Sul (Côrtes et al., 2014a, 2014b). No entanto, é provável que o investimento para a confecção da armadilha não tenha sido feito de uma só vez, mas gradativamente, à medida que ela se mostrou eficiente. Ainda, pode-se mencionar como vantagem da 'ratoeira' em comparação com a 'redinha' o fato de os catadores entrevistados terem afirmado que a primeira possui maior durabilidade, exige menor tempo de manuseio durante e após a captura dos animais, não necessita de lavagem diária e tem menor custo de reparo. Além disso, a armadilha captura os caranguejos-uçá durante períodos em que o catador não está presente no manguezal, permitindo que este se dedique a outras atividades de geração de renda.

A 'ratoeira' captura os caranguejos-uçá vivos e permite que o catador selecione o sexo e o tamanho dos animais que serão comercializados ainda na área de extração, possibilitando a soltura de animais jovens ou de fêmeas ovígeras, por exemplo. Alguns autores afirmam que tal 
seleção também pode ocorrer a partir da utilização da 'redinha' (Côrtes et al., 2014a), enquanto outros afirmam que esta técnica não permite tal seletividade (Nascimento et al., 2011). Como não existe fiscalização frequente, não há garantia de que ocorra a soltura de animais cuja comercialização é proibida. Assim como a 'redinha', a 'ratoeira' é utilizada durante todo ano e não provoca danos físicos aos catadores, diferentemente dos métodos 'braceamento' e 'mão' (ver detalhes em Côrtes et al., 2014b).

Trabalhadores do setor pesqueiro artesanal não são resistentes às mudanças técnicas que envolvem a atividade e, em algumas situações, são obrigados a utilizar métodos considerados como predatórios, a fim de aumentar seu rendimento (Andriguetto-Filho, 2003). A introdução de novos métodos de captura em atividades pesqueiras extrativas pode ocasionar a sobrexplotação das espécies-alvo (Nascimento et al., 2011, 2012), se não houver acompanhamento regular da sua utilização ou caso não sejam adotadas medidas regulatórias adequadas à realidade ambiental e socioeconômica de cada região. Nascimento et al. (2016) relataram que catadores do estuário do rio Mamanguape, estado da Paraíba, estão dispostos a dialogar com órgãos ambientais, a fim de discutir a legislação vigente, que rege as técnicas de coleta do caranguejo-uçá. Esta disponibilidade dos catadores pode estreitar a relação entre os interessados, facilitando, dessa maneira, a incorporação de ações de manejo no cotidiano da extração (Nascimento et al., 2016). A incorporação de novas tecnologias pode induzir à perda de parte do conhecimento tradicional associado à extração do recurso, já que a próxima geração de catadores não precisa se aprofundar no conhecimento sobre a espécie para obter sucesso de coleta (Nascimento et al., 2011).

Desta maneira, métodos não tradicionais de extração do caranguejo-uçá, como a 'ratoeira' e a 'redinha', devem ser utilizados juntamente com estratégias de manejo local, com o intuito de minimizar seus possíveis impactos sobre a população capturada para fins comerciais, tais como a seleção dos animais capturados que serão comercializados, a rotação espacial e/ou temporal das áreas de extração no manguezal e a limitação do esforço de pesca ou de cotas de captura para cada um desses métodos. Ainda, mostra-se importante a participação e a valorização do conhecimento tradicional das comunidades em ações decisivas sobre o uso de recursos de fauna, como o caranguejo-uçá (Alves et al., 2005; Nordi et al., 2009). Portanto, é necessário que ocorra modificação na legislação, a fim de permitir o uso de armadilhas específicas a cada região, bem como deve haver fiscalização, a qual precisa ser educativa e frequente. A junção de uma legislação bem definida, de fiscalização assídua e de ações de manejos local e técnico-científico pode tornar mais efetivas possíveis soluções para evitar a sobrexplotação.

\section{AGRADECIMENTOS}

À presidente da colônia de pescadores Z-1, Diviane Santos das Chagas Barreto, pela anuência para a realização deste estudo; aos catadores de caranguejo-uçá da comunidade de Gargaú, pela concessão das entrevistas; a Sérgio Carvalho Moreira, pela elaboração do mapa. A primeira autora agradece à Coordenação de Aperfeiçoamento de Pessoal de Nível Superior (CAPES), pela concessão de bolsa de doutorado. A segunda autora agradece ao Conselho Nacional de Desenvolvimento Científico e Tecnológico (CNPq) (301.405/13-1) e à Fundação de Amparo à Pesquisa do Estado do Rio de Janeiro (FAPERJ) (E-26/201.161/2014), pelo fomento à pesquisa. A terceira autora agradece ao CNPq (400053/2016-0) e à FAPERJ (E-26/203.202/2016), pelo fomento à pesquisa.

\section{REFERÊNCIAS}

ALENCAR, C. A. G. \& L. P. MAIA, 2011. Perfil socioeconômico dos pescadores brasileiros. Arquivos de Ciências do Mar 44(3): 12-19.

ALVES, R. R. N., 2012. Relationships between fauna and people and the role of ethnozoology in animal conservation. Ethnobiology and Conservation 1(2): 1-69. DOI: <http://dx.doi.org/10.15451/ ec2012-8-1.2-1-69>.

ALVES, R. R. N. \& A. K. NISHIDA, 2002. A ecdise do caranguejouçá, Ucides cordatus l. (Decapoda, Brachyura) na visão dos caranguejeiros. Interciência 27(3): 110-117.

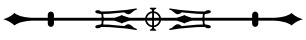


ALVES, R. R. N. \&A. K. NISHIDA, 2003. Aspectos socioeconômicos e percepção ambiental dos catadores de caranguejo-uçá Ucides cordatus cordatus (L. 1763) (Decapoda, Brachyura) do estuário do rio Mamanguape, Nordeste do Brasil. Interciência 28(1): 36-43.

ALVES, R. R. N., A. K. NISHIDA \& M. I. M. HERNANDEZ, 2005. Environmental perception of gatherers of the crab caranguejo-uçá (Ucides cordatus, Decapoda, Brachyura) affecting their collection attitudes. Journal of Ethnobiology and Ethnomedicine 1: 1-8. DOI: <http://dx.doi.org/10.1186/1746-4269-1-10>.

ALVES, R. R. N. \&W. M. S. SOUTO, 2015. Ethnozoology: a brief introduction. Ethnobiology and Conservation 4(1): 1-13. DOI: $<$ http://dx.doi.org/10.15451/ec2015-1-4.1-1-13>.

ANDRIGUETTO-FILHO, J. M., 2003. A mudança técnica e o processo de diferenciação dos sistemas de produção pesqueira do litoral do Paraná, Brasil. Desenvolvimento e Meio Ambiente 8: 43-58. DOI: <https://doi.org/10.5380/dma.v8i0.22050>.

AZEVEDO, C. M. A., 2005. A regulamentação do acesso aos recursos genéticos e aos conhecimentos tradicionais associados no Brasil. Biota Neotropica 5(1): 1-9. Disponível em: $<$ http://www. biotaneotropica.org.br/v5n1/pt/abstract?point-ofview+BN00105012005>. Acesso em: 4 abril 2017.

BAILEY, K. D., 1982. Methods of social research: 2. ed.: 1-553. The Free Press, Macmillan Publishers, Nova York.

BARBOZA, R. S. L., S. NEUMANN-LEITÃO, M. S. L. BARBOZA \& L. M. A. BATISTA-LEITE, 2008. "Fui no mangue catar lixo, pegar caranguejo, conversar com o urubu”: estudo socioeconômico dos catadores de caranguejo no litoral norte de Pernambuco. Revista Brasileira de Engenharia de Pesca 3(2): 117-134. Disponível em: <http://ppg.revistas.uema.br/index.php/REPESCA/article/ view/91/77>. Acesso em: 17 maio 2016.

BERNINI, E., 2008. Estrutura da cobertura vegetal e produção de serapilheira da floresta de mangue do estuário do rio Paraíba do Sul, estado do Rio de Janeiro, Brasil: 1-150. Tese (Doutorado em Ecologia e Recursos Naturais) - Universidade Estadual do Norte Fluminense Darcy Ribeiro, Campos dos Goytacazes.

BOTELHO, E. R. O., M. C. F. SANTOS \& A. C. P. PONTES, 2000. Algumas considerações sobre o uso da redinha na captura do caranguejo-uçá, Ucides cordatus (Linnaeus, 1763), no litoral sul de Pernambuco - Brasil. Boletim Técnico-Científico do CEPENE 8(1): 55-71. Disponível em: <http://www.icmbio.gov.br/cepene/ images/stories/publicacoes/btc/vol08/art03_vol81.pdf > . Acesso em: 10 agosto 2016.

BRASIL, 2003a. Portaria n. 52, de 30 de setembro de 2003. Proíbe, anualmente, a captura, a manutenção em cativeiro, o transporte, o beneficiamento, a industrialização o armazenamento e a comercialização da espécie Ucides cordatus. Diário Oficial da União, 2 outubro 2003. Disponível em: <http://www.ipef. br/legislacao/bdlegislacao/arquivos/18413.rtf > . Acesso em: 10 agosto 2016
BRASIL, 2003b. Portaria n. 53, de 30 de setembro de 2003. Proíbe, anualmente, a captura, a manutenção em cativeiro, o transporte, o beneficiamento, a industrialização o armazenamento e a comercialização da espécie Cardisoma guanhumi. Diário Oficial da União, 2 outubro 2003. Disponível em: <http://wmw.icmbio.gov.br/ portal/publicacoes?showall=\&start=2 > . Acesso em: 10 agosto 2016 .

BRASIL, 2004. Lei n.10.836, de 9 de janeiro de 2004. Cria o Programa Bolsa Família e dá outras providências. Diário Oficial da União, 12 janeiro 2004. Disponível em: < http://www. planalto. gov.br/ccivil_03/_ato2004-2006/2004/lei/110.836.htm>. Acesso em: 10 agosto 2016 .

BRASIL, 2014. Decreto n. 8.232, de 30 de abril de 2014. Altera o decreto n. 5.209, de 17 de setembro de 2004, que regulamenta o Programa Bolsa Família, e o decreto n. 7.492, de 2 de junho de 2011, que institui o Plano Brasil Sem Miséria. Diário Oficial da União, 2 maio 2014. Disponível em: <http://www.planalto.gov.br/ccivil 03/ ato20112014/2014/Decreto/D8232.htm >. Acesso em: 10 agosto 2016.

BRASIL, 2015. Lei n. 13.123, de 20 de maio de 2015. Dispõe sobre o acesso ao patrimônio genético, sobre a proteção e o acesso ao conhecimento tradicional associado e sobre a repartição de benefícios para conservação e uso sustentável da biodiversidade; revoga a Medida Provisória n. 2.186-16, de 23 de agosto de 2001; e dá outras providências. Diário Oficial da União, 14 maio 2015. Disponível em: <http://www.planalto.gov.br/ccivil_03/_Ato20152018/2015/Lei/L13123.htm > . Acesso em: 10 agostō $201 \overline{6}$.

BRASIL, 2016. Decreto n. 8.794, de 29 de junho de 2016. Altera o decreto n. 5.209, de 17 de setembro de 2004, que regulamenta a lei n. 10.836, de 9 de janeiro de 2004, que cria o Programa Bolsa Família, e o decreto n. 7.492, de 2 de junho de 2011, que institui o Plano Brasil Sem Miséria, e dá outras providências. Diário Oficial da União, 30 junho 2016. Disponível em: <http://www.planalto.gov.br/ccivil_03/_ato20152018/2016/decreto/D8794.htm >. Acesso em: 10 agosto 2016.

CAPISTRANO, J. F. \& P. F. M. LOPES, 2012. Crab gatherers perceive concrete changes in the life history traits of Ucides cordatus (Linnaeus, 1763), but overestimate their past and current catches. Ethnobiology and Conservation 1(7): 1-21. DOI: <http://dx.doi.org/10.15451/ ec2012-8-1.7-1-21>.

CARVALHO, H. R. L. \& M. A. IGARASHI, 2009. A utilização do forjo na captura do caranguejo-uçá (Ucides cordatus) na comunidade de Tapebas em Fortaleza - CE. Biotemas 22(1): 69-74. DOI: < https:// doi.org/10.5007/2175-7925.2009v22n1p69>.

ClauzeT, M., M. RAMiRES \& W. A. BARRELLA, 2005. Pesca artesanal e conhecimento local de duas populações caiçaras (enseada do mar virado e barra do una) no litoral de São Paulo, Brasil. Multiciência 4: 1-22.

CÔRTES, L. H. O., C. A. ZAPPES \& A. P. M. DI BENEDITTO, 2014a. Ethnoecology, gathering techniques and traditional management of the crab Ucides cordatus Linnaeus, 1763 in a mangrove forest in south-eastern Brazil. Ocean \& Coastal Management 93: 129-138. DOI: <http://dx.doi.org/10.1016/j.ocecoaman.2014.03.021>.

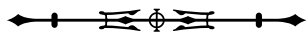


CÔRTES, L. H. O., C. A. ZAPPES \& A. P. M. DI BENEDITTO, 2014b. Extração e cadeia produtiva do caranguejo-uçá no Norte do Rio de Janeiro. Boletim do Instituto de Pesca de São Paulo 40(4): 639-656. Disponível em: <http://www.pesca.sp.gov. $\mathrm{br} / 40$ 4-639-656.pdf>. Acesso em: 31 dezembro 2014.

DIAS-NETO, J. (Org.), 2011. Proposta de Plano Nacional de Gestão para o uso sustentável do caranguejo-uçá, do Guaiamum e do Siri-Azul: 1-156. IBAMA, Brasília.

DIEGUES, A. C., 2000. Etnoconservação: novos rumos para a proteção da natureza nos trópicos: 2. ed.: 1-290. NUPAUB LTDA, São Paulo.

FELLER, I. C., C. E. LOVELOCK, U. BERGER, K. L. MCKEE, S. B. JOYE \& M. C. BALL, 2010. Biocomplexity in mangrove ecosystems. Annual Review of Marine Science 2: 395-417. DOI: <http://dx.doi.org/10.1146/annurev.marine.010908.163809>.

FIRMO, A. M. S., M. M. P. TOGNELLA, S. R. SILVA, R. R. D. BARBOZA \& R. R. N. ALVES, 2012. Capture and commercialization of blue land crabs ("guaiamum") Cardisoma guanhumi (Lattreille, 1825) along the coast of Bahia State, Brazil: an ethnoecological approach. Journal of Ethnobiology and Ethnomedicine 8: 1-12. DOI: < http://dx.doi.org/10.1186/17464269-8-12>.

FisCARELli, A. G. \& M. A. A. PINHEIRO, 2002. Perfil sócioeconômico e conhecimento etnobiológico do catador de caranguejo-uçá, Ucides cordatus (Linnaeus, 1763), nos manguezais de Iguape ( $\left.24^{\circ} 41^{\prime} \mathrm{S}\right)$, SP, Brasil. Actualidades Biologicas 24(77): 39-52. Disponível em: <http://matematicas.udea.edu. $\mathrm{co} / \sim$ actubiol/actualidadesbiologicas/raba2002v24n77art5.pdf $>$. Acesso em: 30 julho 2017.

GIRI, C., E. OCHIENG, L. L. TIESZEN, Z. ZHU, A. SINGH, T. LOVELAND, J. MASEK \& N. DUKE, 2011. Status and distribution of mangrove forests of the world using earth observation satellite data. Global Ecology and Biogeography 20(1): 154-159. DOI: <https://doi.org/10.1111/j.1466-8238.2010.00584.x>.

INSTITUTO BRASILEIRO DE GEOGRAFIA E ESTATÍSTICA (IBGE), 2015. Renda domiciliar per capita, 2015. Disponível em: <http://www.ibge.gov.br/home/estatistica/indicadores/ trabalhoerendimento/pnad_continua/default_renda_percapita. shtm >. Acesso em: 10 julho 2016.

INSTITUTO BRASILEIRO DE GEOGRAFIA E ESTATÍSTICA (IBGE), 2016. Informações sobre os municípios brasileiros, 2016. Disponível em: <http://cidades.ibge.gov.br>. Acesso em: 10 julho 2016.

IVO, C. T. C. \& T. C. V. GESTEIRA, 1999. Sinopse das observações sobre a bioecologia e pesca do caranguejo-uçá, Ucides cordatus cordatus (Linnaeus, 1763), capturado em estuários de sua área de ocorrência no Brasil. Boletim Técnico-Científico do CEPENE 7(1): 9-52.
LIBRETT, M. \& D. PERRONE, 2010. Apples and oranges: ethnography and the IRB. Qualitative Research 10(6): 729747. Disponível em: <http://journals.sagepub.com/doi/ pdf/10.1177/1468794110380548 > . Acesso em: 10 março 2012.

MAGALHÃES, H. F., E. M. COSTA NETO \& A. SCHIAVETTI, 2011. Saberes pesqueiros relacionados à coleta de siris e caranguejos (Decapoda: Brachyura) no município de Conde, Estado da Bahia. Biota Neotropica 11(2): 45-54. DOI: <http://dx.doi.org/10.1590/ S1676-06032011000200005>.

MARTINS, M. C., 2008. Práticas de trabalho e produção de saberes no cotidiano de mulheres pescadoras. Sísifo/Revista de Ciências da Educação (6): 71-84.

NASCIMENTO, D. M., J. S. MOURÃO \& R. R. N. ALVES, 2011. A substituição das técnicas tradicionais de captura do caranguejouçá (Ucides cordatus) pela técnica "redinha" no estuário do rio Mamanguape, Paraíba. Sitientibus série Ciências Biológicas 11(2): 113-119.

NASCIMENTO, D. M., E. N. FERREIRA, D. M. M. S. Q. BEZERRA, P. D. ROCHA, R. R. N. ALVES \& J. S. MOURÃO, 2012. Capture techniques' use of caranguejo-uçá crabs (Ucides cordatus) in Paraíba state (northeastern Brazil) and its socio-environmental implications. Anais da Academia Brasileira de Ciências 84(4): 1051-1064. DOI: <http://dx.doi.org/10.1590/S0001-37652012005000066>.

NASCIMENTO, D. M., A. G. C. ALVES, R. R. N. ALVES, R. R. D. BARBOZA, K. DIELE \& J. S. MOURÃO, 2016. An examination of the techniques used to capture mangrove crabs, Ucides cordatus, in the Mamanguape River estuary, northeastern Brazil, with implications for management. Ocean \& Coastal Management 130: 50-57. DOI: <http://dx.doi.org/10.1016/j. ocecoaman.2016.05.010>.

NASCIMENTO, D. M., R. R. N. ALVES, R. R. D. BARBOZA, A. J. SCHMIDT, K. DIELE \& J. S. MOURÃO, 2017. Commercial relationships between intermediaries and harvesters of the mangrove crab Ucides cordatus (Linnaeus, 1763) in the Mamanguape River estuary, Brazil, and their socio-ecological implications. Ecological Economics 131: 44-51. DOI: <http:// dx.doi.org/10.1016/j.ecolecon.2016.08.017>.

NORDI, N., A. K. NISHIDA \& R. R. N. ALVES, 2009. Effectiveness of two gathering techniques for Ucides cordatus in Northeast Brazil: implications for the sustainability of mangrove ecosystems. Human Ecology 37: 121-127. DOI: <http://dx.doi.org/10.1007/ s10745-009-9214-9>.

OLIVEIRA, P. C., A. P. DI BENEDITTO, E. M. R. BULHÕES \& C. A. ZAPPES, 2016. Artisanal fishery versus port activity in southern Brazil. Ocean \& Coastal Management 129: 149-157. DOI: <http:// dx.doi.org/10.1016/j.ocecoaman.2016.05.005>.

PATTON, M. Q., 1990. Qualitative evaluation and research methods: 2. ed.: 1-532. Sage Publications, Newbury Park. 
SANTA-FÉ, U. M. G. \& A. R. R. ARAÚJO, 2013. Seletividade e eficiência das artes de pesca utilizadas na captura de Ucides cordatus (Linnaeus, 1763), Sergipe, Brasil. Acta of Fisheries and Aquatic Resources 1(1): 29-44. DOI: <https://doi.org/10.2312/ ActaFish.2013.1.1.29-44>.

SILVA, M. C., A. S. OLIVEIRA \& G. Q. NUNES, 2007. Caracterização socioeconômica da pesca artesanal no município de Conceição do Araguaia, estado do Pará. Amazônia: Ciência \& Desenvolvimento 2(4): 37-51.

SPALDING, M. D., M. KANUMA \& L. COLLINS, 2010. World atlas of mangroves: 1. ed.: 1-319. Earthscan, London.

VERGARA FILHO, W. L. \& O. PEREIRA FILHO, 1995. As mulheres do caranguejo. Ecologia e Desenvolvimento 5(53): 34-36.
WALTER, T., J. WILKSON \& P. A. SILVA, 2012. A análise da cadeia produtiva dos catados como subsídio à gestão costeira: as ameaças ao trabalho das mulheres nos manguezais e estuários no Brasil. Revista Gestão Costeira Integrada 12(4): 483-497.

ZAPPES, C. A., P. C. OLIVEIRA \& A. P. M. DI BENEDITTO, 2016. Percepção de pescadores do Norte Fluminense sobre a viabilidade da pesca artesanal com a implantação de megaempreendimento portuário. Boletim do Instituto de Pesca de São Paulo 42(1): $73-$ 88. DOI: <https://doi.org/10.5007/1678-2305.2016v42n1p73>.

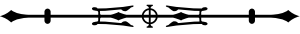


